\title{
Adjuvant postmastectomy radiotherapy might be associated with better survival in women with heart failure receiving total mastectomy
}

\author{
Jiaqiang Zhang ${ }^{1 \dagger}$, Shao-Yin Sum ${ }^{2 \dagger}$, Jeng-Guan Hsu ${ }^{3}$, Ming-Feng Chiang ${ }^{4}$, Tian-Shyug Lee ${ }^{3}$ and
} Szu-Yuan $\mathrm{Wu}^{3,5,6,7,7,8,9,10^{*}}$ (1)

\begin{abstract}
Background: To date, no data on the effect of adjuvant postmastectomy radiotherapy (PMRT) on oncologic outcomes, such as all-cause death, locoregional recurrence (LRR), and distant metastasis (DM), are available in women with left-side breast invasive ductal carcinoma (IDC) and heart failure with reduced ejection fraction (HFrEF).
\end{abstract}

Patients and methods: We enrolled 646 women with left-breast IDC at clinical stages I-IIIC and HFrEF receiving radical total mastectomy (TM) followed by adjuvant PMRT or non-adjuvant PMRT. We categorized them into two groups based on their adjuvant PMRT status and compared their overall survival (OS), LRR, and DM outcomes. We calculated the propensity score and applied inverse probability of treatment weighting (IPTW) to create a pseudo-study cohort. Furthermore, we performed a multivariate analysis of the propensity score-weighted population to obtain hazard ratios (HRs).

Results: In the IPTW-adjusted model, adjuvant PMRT (adjusted HR [aHR]: 0.52; 95\% confidence interval [CI]: 0.370.74 ) was a significant independent prognostic factor for all-cause death $(P=0.0003)$, and the aHR $(95 \% \mathrm{Cl})$ of $\mathrm{LRR}$ and DM for adjuvant PMRT was $0.90(0.79-0.96 ; P=0.0356)$ and $0.89(0.54-1.50 ; P=0.6854)$, respectively, compared with the nonadjuvant PMRT group.

Conclusion: Adjuvant PMRT was associated with a decrease in all-cause death, and LRR in women with left IDC and HFrEF compared with nonadjuvant PMRT.

Keywords: Breast cancer, Radiation-induced cardiovascular toxicity, Total mastectomy, Radiotherapy, Survival

*Correspondence: szuyuanwu5399@gmail.com

${ }^{\dagger}$ Jiaqiang Zhang and Shao-Yin Sum have contributed equally to this study

${ }^{7}$ Division of Radiation Oncology, Lo-Hsu Medical Foundation, Lotung

Poh-Ai Hospital, No. 83, Nanchang St., Luodong Township, Yilan County 265, Taiwan

Full list of author information is available at the end of the article

\section{Key points}

- Question: Is adjuvant postmastectomy radiotherapy (PMRT) worthy for women with left-side breast invasive ductal carcinoma (IDC) and heart failure with reduced ejection fraction (HFrEF) receiving total mastectomy (TM)?

- Findings: In the IPTW-adjusted models, adjuvant PMRT was associated with a decrease in all-cause 
death and LRR in women with left IDC and HFrEF compared with no adjuvant PMRT.

- Meaning: We suggest adjuvant PMRT for women with left-side IDC receiving TM, even when they have HFrEF.

\section{Introduction}

Radiation-induced cardiovascular toxicity (RICT) is associated with a portion of the heart being placed in a radiation field [1]. For patients with left-sided breast cancers, careful treatment planning and usage of contemporary radiotherapy (RT) techniques are critical to minimize cardiac exposure to radiation [1]. Incidental irradiation dose to the heart as part of the initial treatment for breast cancer can result in a range of cardiotoxic effects, including coronary artery disease (CAD), cardiomyopathy, pericardial disease, valvular dysfunction, and conduction abnormalities [2-4]. At present, no recommended minimum radiation dose that is completely safe exists [3].

The association of RICT is not dependent on the presence or absence of a breast but on the volume of radiation to the heart $[3,4]$. Thus, cardiotoxicities associated with RT differ between the postlumpectomy and postmastectomy settings; this is because in the postmastectomy setting, the RT field often includes the nodal tissues, and these nodes are not always targeted in the postlumpectomy setting $[5,6]$. Thus, postmastectomy RT (PMRT) is more often associated with cardiac disease relative to postlumpectomy $\mathrm{RT}$, but this is likely a result of the usually larger irradiated volumes of the heart in postmastectomy RT $[5,6]$. Therefore, RICT in patients with breast cancer should be separately discussed by using different surgical techniques of breast-conserving surgery (BCS) and total mastectomy (TM). Hereby, we wanted to address the values of adjuvant PMRT for breast cancer patients with heart failure (HF) receiving TM with large RT field and high dose-volume to the normal heart.

The crucial issue is whether adjuvant PMRT can be safely given to women with HF and left-side breast cancer who receive TM. No data are available to address the value of adjuvant PMRT in women with breast cancer and HF receiving TM. HF due to left ventricle (LV) dysfunction is categorized according to LV ejection fraction (LVEF) as HF with reduced ejection fraction (LVEF $\leq 40 \%$, known as HFrEF) [7-9]. Until now, no study has estimated the oncologic outcomes of adjuvant PMRT in women with breast invasive ductal carcinoma (IDC) and HFrEF receiving TM.

\section{Patients and methods}

\section{Study population}

In this cohort study, data were retrieved from the Taiwan Cancer Registry Database (TCRD). We enrolled women with $\mathrm{HF}$ with reduced ejection fraction $(\mathrm{LVEF} \leq 40 \%$; HFrEF) [7-9] who had received a diagnosis of left-side breast IDC between January 1, 2008, and December 31,2018 . The index date was the date of TM, and the follow-up duration was from the index date to December 31, 2019. The TCRD of the Collaboration Center of Health Information Application contains detailed cancerrelated information of patients, including their clinical stage, pathologic stages, chemotherapy regimens, dose of chemotherapy, molecular status, drug use, hormone receptor status, radiation modalities and doses, and surgical procedures [10-13]. The study protocols were reviewed and approved by the Institutional Review Board of Tzu-Chi Medical Foundation (IRB109-015-B).

\section{Inclusion and exclusion criteria}

The diagnoses of the enrolled patients with HFrEF were confirmed after their pathological data were reviewed, and the women with newly diagnosed left-side IDC were confirmed to have no other cancers or distant metastases. The women with HFrEF were included if they had received a left-side IDC diagnosis, were 20 years old or older, and had clinical stage I-IIIC (American Joint Committee on Cancer [AJCC], 8th edition) without metastasis. Patients with HFrEF were excluded if they had a history of cancer before the IDC diagnosis date, unknown pathologic types, missing sex data, unclear staging, or non-IDC histology. In addition, patients with unclear differentiation of tumor grade, missing HR status, missing data on hormone therapy or trastuzumab use, or unclear staging were excluded. Adjuvant treatments such as adjuvant chemotherapy, hormone therapy, or the trastuzumab use did not constitute exclusion criteria based on the National Comprehensive Cancer Network (NCCN) guidelines [14]. We also excluded patients with HFrEF with unclear data on surgical procedures such as TM or TM, ill-defined nodal surgery, unclear Charlson comorbidity index (CCI), or unclear differentiation from our cohort. Hormone receptor positivity was defined as $\geq 1 \%$ of tumor cells demonstrating positive nuclear staining through immunohistochemistry [15].

After applying the inclusion and exclusion criteria, we enrolled 646 women with HFrEF and AJCC clinical stage I-IIIC and left-side IDC who had received a TM followed by sentinel lymph node biopsy (SLNB) or axillary lymph node dissection (ALND) and divided them into two groups based on their adjuvant PMRT status to compare all-cause mortality: Group 1 (women with left-side 
IDC and HFrEF who received TM followed by adjuvant PMRT) and Group 2 (women with left-side IDC and HFrEF who received TM and no adjuvant PMRT). We also excluded women in Group 1 receiving nonstandard adjuvant PMRT. Standard postmastectomy RT is irradiation to both the chest wall and to the regional nodes with 50 Gy at least. These include the supraclavicular and infraclavicular nodes. We also include RT to the axilla except in some patients who underwent complete axillary dissection. Contemporary RT techniques were included in our study (intensity modulated radiation therapy [IMRT] and volumetric modulated arc therapy [VMAT]) and the conventional two-dimensional RT technique was excluded. The included contemporary RT techniques were three-dimensional RT and intensity-modulated radiation therapy. The incidence of comorbidities was scored using the CCI $[16,17]$. Hypertension, diabetes, and coronary arterial diseases (CAD) were excluded from the CCI scores to avoid repetitive adjustment in multivariate analysis. Only comorbidities observed within 6 months before the index date were included; they were coded and classified according to the International Classification of Diseases, 10th Revision, Clinical Modification (ICD-10-CM) codes at the first admission or based on more than two repetitions of a code issued at outpatient department visits.

\section{Study covariates and statistical analysis}

Significant independent predictors, namely age, diagnosis year, CCI score, differentiation, $\mathrm{pT}$, $\mathrm{pN}$, hypertension, $\mathrm{CAD}$, diabetes, chemotherapy with anthracycline-based regimen, hormone receptor status, trastuzumab use, nodal surgery, and hospital level (academic or nonacademic), were analyzed using a multivariate analysis of the propensity score-weighted population to determine hazard ratios (HRs). We calculated the propensity score and applied inverse probability of treatment weighting (IPTW) to create a pseudo-study cohort; the weighted cohort avoids covariate bias and mimics randomized adjuvant PMRT or no adjuvant PMRT assignment: IPTW for patients with PMRT $=1 / \mathrm{p}(\mathrm{PMRT})$; IPTW for patients without $\mathrm{PMRT}=1 /(1-\mathrm{p}[\mathrm{PMRT}])[18,19]$. The independent predictors were examined in multivariable analyses after IPTW adjustment. Moreover, they were controlled for and were stratified in the analysis. The endpoint was all-cause death in the women with left-side IDC and HFrEF who received TM followed by adjuvant PMRT (Group 1, case group) and in the women with left IDC and HFrEF who received TM and had no adjuvant PMRT (Group 2, control group).

The cumulative incidence of death was estimated using the Kaplan-Meier method, and differences in the overall survival (OS), locoregional recurrence
(LRR)-free survival, and distant metastasis (DM)-free survival between women with left IDC and HFrEF receiving TM followed by adjuvant PMRT versus no adjuvant PMRT were determined using a log-rank test. After confounders were adjusted for, IPTW-adjusted models were used to determine the time from the index date to all-cause mortality in the women with left IDC and HFrEF who received TM followed by adjuvant PMRT or no adjuvant PMRT. Subsequently, in a multivariate analysis, HRs were adjusted for age, diagnosis year, CCI scores, differentiation, $\mathrm{pT}$, pN, hypertension, CAD, diabetes, chemotherapy with anthracycline, hormone receptor status, trastuzumab use, nodal surgery, and hospital levels. All analyses were conducted using SAS (Version 9.4; SAS, Cary, NC, USA), and a two-tailed $P$ value $<0.05$ was considered statistically significant.

\section{Results}

Study cohort

We enrolled 646 women with left-breast IDC at clinical stages I-IIIC and HFrEF who received TM followed by adjuvant PMRT or no adjuvant PMRT (Table 1). Among these women, 143 with left IDC and HFrEF received TM followed by adjuvant PMRT (Group 1) and 503 with left IDC and HFrEF received TM with no adjuvant PMRT (Group 2). After IPTW was executed using the propensity score, the covariates between Groups 1 and 2 were found to be homogenous. The median follow-up durations after the index date were 6.96 and 5.09 years for women with left IDC and HFrEF who received TM followed by adjuvant PMRT or no adjuvant PMRT, respectively. All standardized differences in covariates were smaller than 0.1 (Table 1) and were homogenous between the two groups [20].

\section{Effects of adjuvant PMRT on oncologic outcomes in women with left-side IDC and HFrEF receiving TM}

IPTW-adjusted models indicated that adjuvant PMRT was a significantly better independent prognostic factor for OS, and LRR in the women with left IDC and HFrEF receiving TM (Table 2). Adjuvant PMRT (adjusted HR [aHR]: 0.52; 95\% confidence interval [CI]: 0.37-0.74) was a significant independent prognostic factor for all-cause death $(P=0.0003$; Table 2$)$. In the IPTW-adjusted model, the aHR $(95 \% \mathrm{CI})$ for LRR in the adjuvant PMRT group was $0.90(0.79-0.96 ; P=0.0356$; Table 2) compared with the no adjuvant PMRT group. Moreover, the aHR (95\% CIs) for DM in the adjuvant PMRT group was 0.89 (0.54$1.50 ; P=0.6854)$ compared with the no adjuvant PMRT group (Table 2). 
Table 1 Demographics of patients with breast cancer and heart failure with reduced ejection fraction who received total mastectomy in the propensity score-weighted population through inverse probability of treatment weighting

\begin{tabular}{|c|c|c|c|c|c|}
\hline & \multicolumn{5}{|c|}{ Propensity score weighting population } \\
\hline & \multicolumn{2}{|c|}{$\begin{array}{l}\text { Adjuvant RT } \\
\mathrm{N}=143\end{array}$} & \multicolumn{2}{|c|}{$\begin{array}{l}\text { Non-RT } \\
N=503\end{array}$} & \multirow[t]{2}{*}{$\begin{array}{l}\text { Standardized } \\
\text { difference }\end{array}$} \\
\hline & $\mathrm{n}$ & (\%) & $n$ & $(\%)$ & \\
\hline \multicolumn{6}{|l|}{ Age } \\
\hline Mean (SD) & 67.5 & $(11.3)$ & 67.7 & $(11.2)$ & 0.0215 \\
\hline Median (Q1-Q3) & 67 & $(57-72)$ & 67 & $(61-77)$ & \\
\hline $20-69$ & 67 & $(46.9)$ & 238 & $(47.9)$ & 0.0096 \\
\hline $70+$ & 76 & $(53.1)$ & 259 & $(52.1)$ & \\
\hline \multicolumn{6}{|l|}{ Diagnosis year } \\
\hline 2009-2012 & 88 & $(50.9)$ & 250 & $(50.3)$ & 0.0115 \\
\hline $2013-2016$ & 85 & $(49.1)$ & 247 & $(49.7)$ & \\
\hline \multicolumn{6}{|l|}{$\mathrm{CCl}$ scores } \\
\hline 0 & 44 & $(30.8)$ & 123 & $(24.5)$ & 0.1433 \\
\hline $1+$ & 99 & $(69.2)$ & 380 & $(75.5)$ & \\
\hline \multicolumn{6}{|l|}{ Differentiation } \\
\hline 1 & 20 & $(14.0)$ & 72 & $(14.3)$ & 0.0212 \\
\hline$\|$ & 77 & $(53.8)$ & 273 & $(54.3)$ & 0.0311 \\
\hline III & 46 & $(32.1)$ & 158 & $(31.4)$ & 0.0207 \\
\hline \multicolumn{6}{|l|}{ AJCC pathologic stage } \\
\hline$\|$ & 17 & $(12.8)$ & 61 & $(12.1)$ & 0.0114 \\
\hline IIIA & 68 & $(47.6)$ & 241 & $(47.9)$ & 0.0162 \\
\hline$\| \mathrm{IIB}-\mathrm{C}$ & 58 & $(40.6)$ & 201 & $(40.0)$ & 0.0142 \\
\hline \multicolumn{6}{|l|}{ pT } \\
\hline pT1 & 9 & $(6.3)$ & 30 & $(5.9)$ & 0.0238 \\
\hline pT2 & 58 & $(40.6)$ & 202 & $(40.2)$ & 0.0149 \\
\hline pT3-4 & 76 & $(53.1)$ & 271 & $(53.9)$ & 0.0130 \\
\hline \multicolumn{6}{|l|}{$\mathrm{pN}$} \\
\hline pNO & 57 & (39.8) & 200 & (39.8) & 0.0000 \\
\hline pN1 & 43 & $(30.1)$ & 151 & $(30.0)$ & 0.0013 \\
\hline pN2-3 & 43 & $(30.1)$ & 152 & $(30.2)$ & 0.0001 \\
\hline Hypertension & 110 & $(76.9)$ & 387 & $(76.9)$ & 0.0001 \\
\hline CAD & 53 & $(37.1)$ & 184 & (36.6) & 0.0092 \\
\hline Diabetes & 60 & $(42.0)$ & 215 & $(42.7)$ & 0.0097 \\
\hline Anthracycline-based chemotherapy & 76 & $(53.1)$ & 260 & $(51.7)$ & 0.0280 \\
\hline Hormone receptor positive & 59 & $(41.3)$ & 229 & $(45.5)$ & 0.0934 \\
\hline Trastuzumab use & 69 & $(48.3)$ & 246 & $(48.9)$ & 0.0031 \\
\hline \multicolumn{6}{|l|}{ Nodal surgery } \\
\hline ALND & 87 & $(60.8)$ & 302 & $(60.0)$ & 0.0079 \\
\hline SLNB & 56 & $(39.2)$ & 201 & $(40.0)$ & \\
\hline \multicolumn{6}{|l|}{ Hospital level } \\
\hline Academic center & 80 & $(55.9)$ & 285 & $(56.7)$ & 0.0181 \\
\hline Nonacademic center & 63 & $(44.1)$ & 218 & $(43.3)$ & \\
\hline
\end{tabular}

$R T$ radiotherapy, IQR interquartile range, SD standard deviation, AJCC American Joint Committee on Cancer, HER2 human epidermal growth factor receptor-2, CCI Charlson comorbidity index, $T$ tumor, $N$ nodal, $p T$ pathologic tumor stage, $p N$ pathologic nodal stage, ALND axillary lymph node dissection, SNLB sentinel lymph node biopsy, $C A D$ coronary arterial disease 
Table 2 Multivariate analysis of propensity score-weighted population with breast cancer and heart failure with reduced ejection fraction receiving total mastectomy

\begin{tabular}{|c|c|c|c|c|c|c|c|c|c|}
\hline & \multicolumn{3}{|l|}{ Death } & \multicolumn{3}{|c|}{ Local recurrence } & \multicolumn{3}{|c|}{ Distant metastasis } \\
\hline & $\mathrm{aHR}^{*}$ & $(95 \% \mathrm{Cl})$ & $P$ value & $\mathrm{aHR}^{*}$ & $(95 \% \mathrm{Cl})$ & $P$ value & $\mathrm{aHR}^{*}$ & $(95 \% \mathrm{Cl})$ & $p$ value \\
\hline \multicolumn{10}{|l|}{ Adjuvant RT } \\
\hline No & Ref & & 0.0003 & Ref & & 0.0356 & Ref & & 0.6854 \\
\hline Yes & 0.52 & $(0.37-0.74)$ & & 0.90 & $(0.79-0.96)$ & & 0.89 & $(0.54-1.50)$ & \\
\hline \multicolumn{10}{|l|}{ Age } \\
\hline 20-69 & Ref & & 0.0020 & Ref & & 0.1200 & Ref & & 0.2901 \\
\hline $70+$ & 1.63 & $(1.20-2.22)$ & & 1.29 & $(0.81-2.49)$ & & 0.75 & $(0.44-1.28)$ & \\
\hline \multicolumn{10}{|l|}{ Diagnosis year } \\
\hline 2009-2012 & Ref & & 0.3507 & Ref & & 0.2770 & Ref & & 0.7421 \\
\hline 2013-2016 & 0.79 & $(0.69-1.31)$ & & 0.73 & $(0.58-1.21)$ & & 0.81 & $(0.57-1.75)$ & \\
\hline \multicolumn{10}{|l|}{ CCl scores } \\
\hline 0 & Ref & & 0.0322 & Ref & & 0.1434 & Ref & & 0.2112 \\
\hline 1 & 1.26 & $(1.14-1.97)$ & & 1.21 & $(0.92-1.79)$ & & 1.35 & $(0.85-1.97)$ & \\
\hline \multicolumn{10}{|l|}{ Differentiation } \\
\hline I & Ref & & 0.0177 & Ref & & 0.0146 & Ref & & 0.0046 \\
\hline$\|$ & 1.09 & $(1.01-1.60)$ & & 1.36 & $(1.02-3.59)$ & & 1.36 & $(1.02-3.59)$ & \\
\hline III & 1.47 & $(1.09-2.40)$ & & 1.37 & $(1.11-3.71)$ & & 1.37 & $(1.01-3.71)$ & \\
\hline \multicolumn{10}{|l|}{ pT } \\
\hline pT1 & Ref & & $<0.0001$ & Ref & & 0.0016 & Ref & & 0.0196 \\
\hline pT2 & 1.38 & $(1.07-1.97)$ & & 1.35 & $(1.05-3.12)$ & & 1.09 & $(1.04-3.04)$ & \\
\hline pT3-4 & 2.91 & $(1.90-4.44)$ & & 2.62 & $(1.19-4.72)$ & & 2.35 & $(1.13-4.89)$ & \\
\hline \multicolumn{10}{|l|}{$\mathrm{pN}$} \\
\hline pNO & Ref & & $<0.0001$ & Ref & & 0.0040 & Ref & & 0.0082 \\
\hline pN1 & 1.94 & $(1.38-2.72)$ & & 1.09 & $(1.03-1.41)$ & & 2.38 & $(1.37-4.12)$ & \\
\hline pN2-3 & 2.90 & $(2.01-4.18)$ & & 1.26 & $(1.06-1.37)$ & & 1.88 & $(1.01-3.51)$ & \\
\hline Hypertension & 1.08 & $(0.77-1.81)$ & 0.4882 & 0.90 & $(0.59-1.56)$ & 0.7217 & 0.95 & $(0.69-1.48)$ & 0.8021 \\
\hline CAD & 1.11 & $(0.71-1.92)$ & 0.3427 & 0.84 & $(0.53-1.39)$ & 0.6914 & 0.94 & $(0.78-1.59)$ & 0.3426 \\
\hline Diabetes & 1.11 & $(0.73-1.90)$ & 0.3422 & 1.01 & $(0.70-1.51)$ & 0.4521 & 0.90 & $(0.55-1.91)$ & 0.8909 \\
\hline Hormone receptor positive & 0.87 & $(0.80-0.91)$ & 0.0312 & 0.77 & $(0.45-0.82)$ & 0.0204 & 0.72 & $(0.63-0.97)$ & 0.0322 \\
\hline Trastuzumab use & 1.07 & $(0.87-1.42)$ & 0.34661 & 1.09 & $(0.58-2.01)$ & 0.3831 & 1.06 & $(0.81-1.54)$ & 0.3421 \\
\hline Anthracycline-based chemotherapy & 0.94 & $(0.57-1.88)$ & 0.4065 & 0.93 & $(0.78-1.83)$ & 0.2412 & 0.84 & $(0.70-2.20)$ & 0.1683 \\
\hline \multicolumn{10}{|l|}{ Nodal surgery } \\
\hline ALND & Ref & & 0.3322 & Ref & & 0.2434 & Ref & & 0.2112 \\
\hline SLNB & 1.06 & $(0.54-1.09)$ & & 1.01 & $(0.82-1.79)$ & & 1.15 & $(0.85-2.97)$ & \\
\hline \multicolumn{10}{|l|}{ Hospital level } \\
\hline Academic center & Ref & & 0.2177 & Ref & & 0.2177 & Ref & & 0.8146 \\
\hline Nonacademic center & 0.99 & $(0.61-1.60)$ & & 0.99 & $(0.61-1.60)$ & & 1.36 & $(0.52-3.59)$ & \\
\hline
\end{tabular}

aHR adjusted hazard ratios, $C l s$ confidence intervals, HR hormone receptor, Her-2 human epidermal growth factor receptor-2, $C C I C h a r l s o n$ comorbidity index, $T$ tumor, $N$ nodal, $p T$ pathologic tumor stage, $p N$ pathologic nodal stage, $A L N D$ axillary lymph node dissection, SNLB sentinel lymph node biopsy, ref reference group, $R T$ radiotherapy

*All covariates mentioned in Table were adjusted

Other independent predictors of all-cause death, LRR, and DM in the women with left IDC and HFrEF receiving TM Old age ( $>70$ years), $C C I \geq 1$, advanced $\mathrm{pT}$ stages (pT2-4), advanced $\mathrm{pN}$ stages (pN1-3), hormone receptor negative status, and differentiation Grade II and III were identified as crucial independent poor prognostic factors for OS (Table 2). IPTW-adjusted models were adjusted for age, diagnosis year, CCI score, differentiation, $\mathrm{pT}, \mathrm{pN}$, hypertension, CAD, diabetes, chemotherapy with anthracycline-based regimen, hormone receptor status, trastuzumab use, nodal surgery, and hospital levels; the aHRs (95\% CIs) 


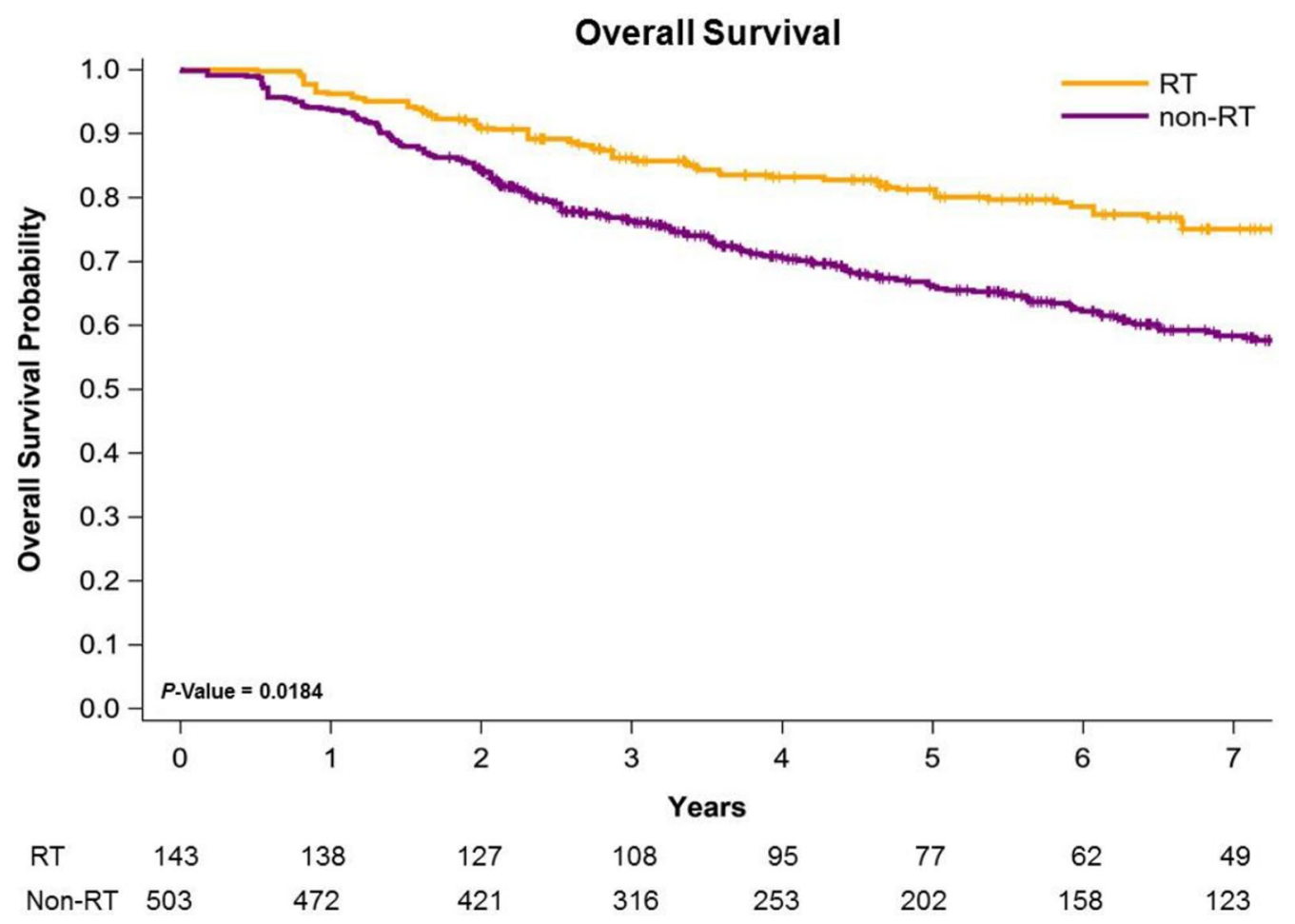

Fig. 1 Kaplan-Meier overall survival curves of propensity score-weighted population with breast cancer and heart failure with reduced ejection fraction receiving total mastectomy

of all-cause death for age $>70$ years, CCI $\geq 1$, differentiation Grades II and III, pT2, pT3-4, pN1, pN23 , and hormone receptor positive status were 1.63 (1.20-2.22), 1.26 (1.14-1.97), 1.09 (1.01-1.60), 1.47 (1.09-2.40), 1.38 (1.07-1.97), 2.91 (1.90-4.44), 1.94 (1.38-2.72), 2.90 (2.01-4.18), and 0.87 (0.80-0.91) compared with age 20-70 years, $\mathrm{CCI}=0$, differentiation grade I, pT1, pN0, and hormone receptor negative status, respectively (Table 2). IPTW-adjusted models also revealed the aHRs (95\% CIs) of LRR for differentiation grade II, differentiation grade III, pT2, pT3-4, $\mathrm{pN} 1, \mathrm{pN} 2-3$, and hormone receptor positive status to be 1.36 (1.02-3.59), 1.37 (1.11-3.71), 1.35 (1.05-3.12), 2.62 (1.19-4.72), 1.09 (1.03-1.41), $1.261 .06-1.37)$, and $0.77(0.45-0.82)$ compared with differentiation grade I, pT1, pN0, and hormone receptor negative status, respectively. Moreover, the aHRs (95\% CIs) of DM for differentiation grade II, differentiation grade III, pT2, pT3-4, pN1, pN2-3, and hormone receptor positive status were 1.36 (1.02-3.59), 1.37 (1.01-3.71), 1.09 (1.04-3.04), 2.35 (1.13-4.89), 2.38 (1.37-4.12), 1.88 (1.01-3.51), and $0.72(0.63-0.97)$ compared with differentiation grade I, pT1, pN0, and hormone receptor negative status, respectively.

\section{Survival curves of adjuvant PMRT or no adjuvant PMRT} in women with left IDC and HFrEF receiving TM

Figure 1 presents Kaplan-Meier curves that illustrate the OS of the women with left IDC and HFrEF receiving TM with adjuvant PMRT or no adjuvant PMRT. The 5-year overall survival rates were $86.01 \%$ and $67.32 \%$ in the adjuvant PMRT and no adjuvant PMRT groups, respectively (Fig. 1); the OS rate was associated with an increasing in the adjuvant PMRT group (log-rank test, $P=0.0184$ ) compared with the non-RT group. Additionally, the 5-year LRR-free survival in women with left IDC and HFrEF receiving TM was $88.43 \%$ and $73.65 \%$ in the adjuvant PMRT group and no adjuvant PMRT group, respectively (Fig. 2; log-rank test, $P=0.0319$ ). The 5 -year DM-free survival in women with left IDC and HFrEF receiving TM was $84.43 \%$ and $86.91 \%$ in the adjuvant PMRT group and no adjuvant PMRT group, respectively (Fig. 3; log-rank test, $P=0.8577$ ).

\section{Discussion}

PMRT has two potential benefits: one is a decrease in the rate of locoregional recurrence, and another is an increase in long-term breast cancer-specific and overall survivals for certain patient populations. These benefits have been consistently reported in multiple studies 


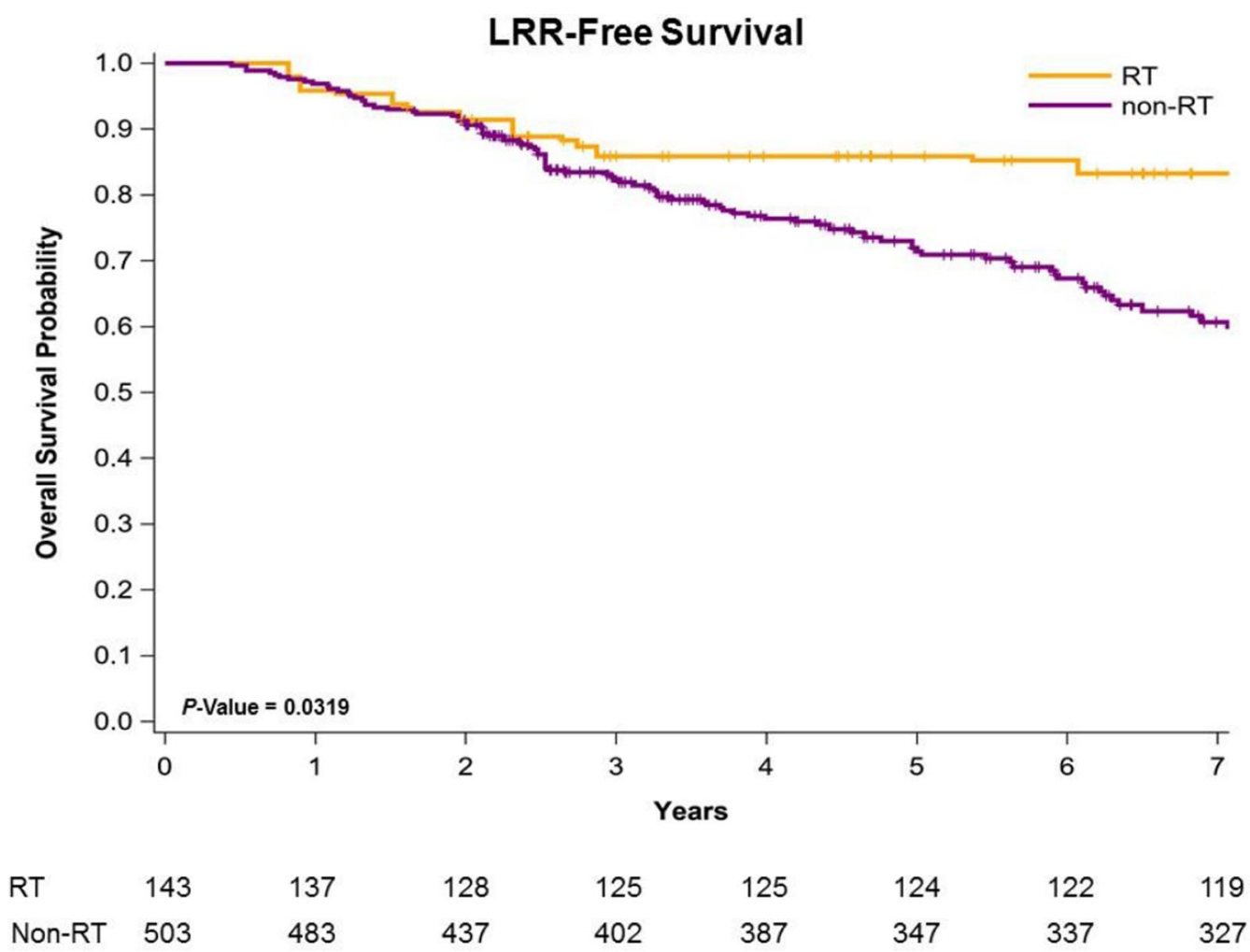

Fig. 2 Kaplan-Meier locoregional recurrence-free survival curves of propensity score-weighted population with breast cancer and heart failure with reduced ejection fraction receiving total mastectomy

[21-23]. Decisions on who should receive PMRT depend on the baseline risk for recurrence. The success of RT, used either alone or in combination with other modalities, has resulted in large cohorts of breast cancer survivors who are vulnerable to late complications such as RICT from RT [5, 24-30]. Numerous treatment-related factors are responsible for cardiotoxicity in women with breast cancer [31-41]. Thus, we conducted the study to determine the survival benefits offered by adjuvant PMRT in women with left-side IDC and HFrEF receiving TM.

Patients with breast cancer might experience adverse effects from many cardiotoxic treatments such as adjuvant PMRT, anthracycline-based chemotherapy, or trastuzumab [5, 6, 24-41]. Although cardiovascular diseases such as HF, heart attacks, and stroke remain the leading cause of death in women, many believe breast cancer to be more deadly [42]. In fact, the risk of RICT should be weighed against the potential benefits of adjuvant PMRT with respect to the patients' prognosis and likely clinical benefit [5, 24-30]. Until now, no data have been available for the evaluation of oncologic outcomes (OS, LRR, and DM) of adjuvant PMRT in women with left-side breast IDC and HFrEF receiving TM. This is the first study to explore the value of adjuvant PMRT for women with leftside breast IDC and HFrEF receiving TM. As shown in Table 2, adjuvant PMRT resulted in better OS, and LRRfree status compared with no adjuvant PMRT in women with left-side breast IDC and HFrEF receiving TM. The potential reasons might be the recent decline in mortality in women with HF $[43,44]$ and the advances in contemporary RT techniques with reduced irradiation volumes to the heart $[2,26,27]$.

According to our literature review, this is the first study to estimate the oncologic outcomes of adjuvant PMRT among women with left-side breast IDC and HFrEF receiving TM. No consensus or evidence for the use of adjuvant PMRT in women with left-side breast IDC and HFrEF receiving TM is present. In the IPTW-adjusted models, adjuvant PMRT was associated with a decrease in the risk of all-cause death, and LRR among women with left-side breast IDC and HFrEF receiving TM (Table 2). The improvement in contemporary RT techniques with decreased irradiation doses and decreased volumes to the heart and the long-term improvement in mortality rates in patients with HFrEF over time might have caused the significant beneficial oncologic outcomes of adjuvant PMRT in women with left-side breast 


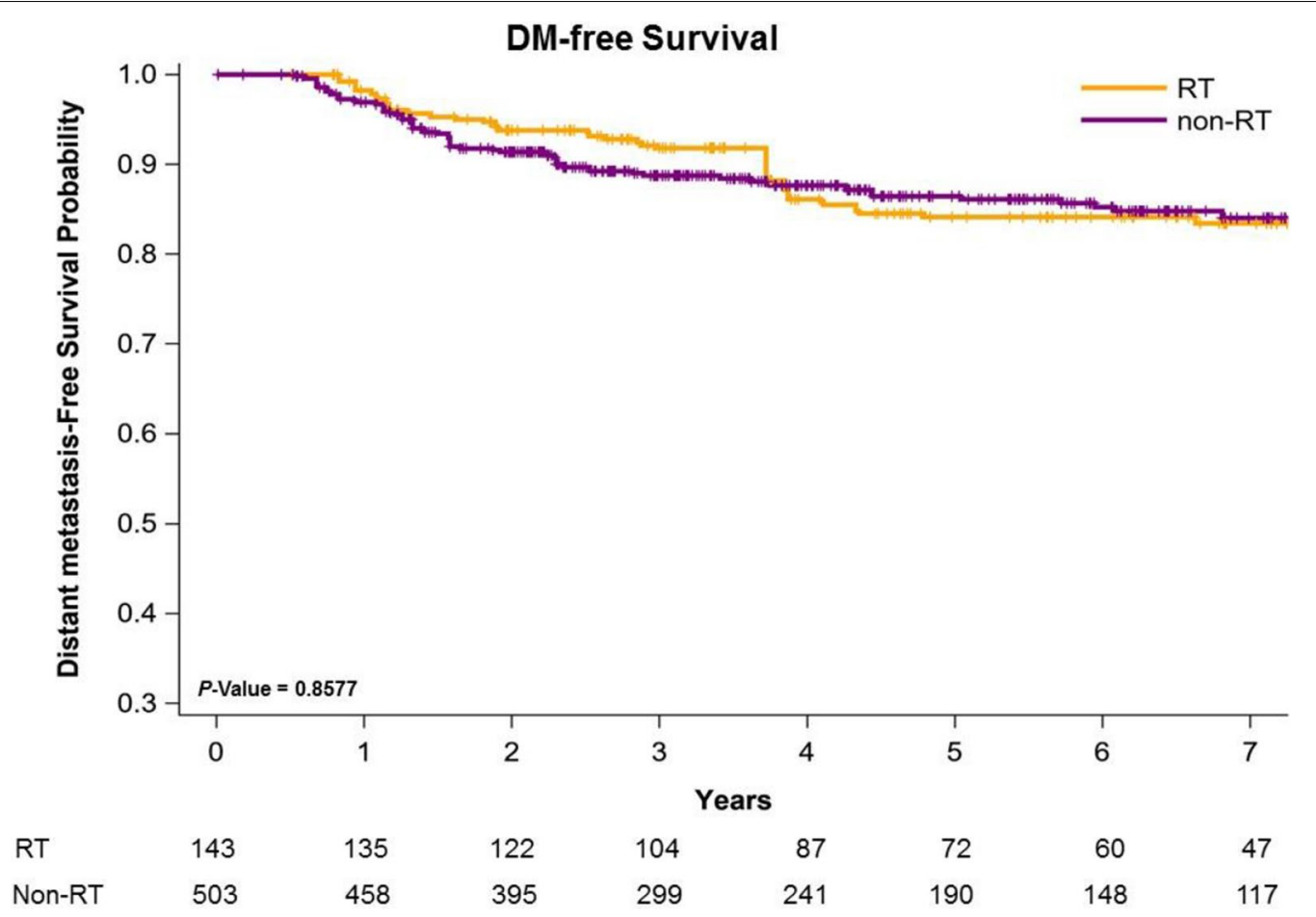

Fig. 3 Kaplan-Meier distant metastasis-free survival curves of propensity score-weighted population with breast cancer and heart failure with reduced ejection fraction receiving total mastectomy

IDC and HFrEF receiving TM [2, 26, 27]. Our study is the first to demonstrate that the potential benefits of adjuvant PMRT with contemporary RT techniques outweigh the risk of RICT given the patients' prognosis and likely long-term OS and LRR benefits (Table 2). According to our findings, we strongly suggested that women with left-side breast IDC and HFrEF receiving TM should also receive adjuvant PMRT to decrease the risk of all-cause death, and LRR.

As shown in Table 2, adjuvant PMRT was a significant prognostic factor for OS and LRR compared with no adjuvant PMRT in women with left-side IDC and HFrEF receiving $\mathrm{TM}$; moreover, old age ( $>70$ years), $\mathrm{CCI} \geq 1$, advanced pT stages (pT2-4), advanced $\mathrm{pN}$ stages (pN13 ), hormone receptor negative status, and differentiation Grade II-III were significant prognostic factors for OS, compatible with findings of previous studies $[10,11$, 45-52]. Moreover, advanced pT stages (pT2-4), pN stages (pN1-3), hormone receptor negative status, and differentiation Grade II-III were significant poor prognostic factors for LRR and DM in women with left-side breast IDC and HFrEF receiving TM, which is also compatible with findings of previous studies [10, 11, 45-52]. Our findings of prognostic factors for OS, LRR, and DM in women with IDC and HFrEF receiving TM are similar with those of previous studies [10,11,45-52], and no additional prognostic factor has been identified in previous studies other than the ones determined in the current study irrespective of whether underlying HFrEF was present.

The potential reasons of better oncologic outcomes on adjuvant PMRT for breast IDC with HFrEF might be attributed to the modern RT techniques. The use of modern RT techniques (such as IMRT and VMAT) as well as the reduction of treatment volumes (partial breast irradiation) allow to reduce acute and late side effects [53-55]. The contemporary RT techniques allow more precise RT field to target volume and decrease RT dose-volume to heart contributed to less RICT $[53,54]$. Therefore, breast IDC patients with HFrEF receiving TM and adjuvant PMRT could get benefits from PMRT and less acute and late toxicity to heart contributed to better oncologic outcomes like OS and LRR-free survival (Table 2, Figs. 1, 2).

A strength of our study was that it was the first cohort study to estimate the survival outcomes of adjuvant PMRT or no adjuvant PMRT among women with left-side IDC and HFrEF receiving TM. The covariates between the adjuvant PMRT and no adjuvant PMRT groups were homogenous for women with left-side IDC and HFrEF receiving TM, with no selection bias (Table 1). No study has estimated the effect of adjuvant PMRT on women with left-side IDC and HFrEF receiving TM. In our study, the poor prognostic factors for OS in women 
with left-side IDC and HFrEF receiving TM were old age, $\mathrm{CCI} \geq 1$, advanced $\mathrm{pT}$ stages (pT2-4), advanced $\mathrm{pN}$ stages ( $\mathrm{pN} 1-3)$, hormone receptor negative status, and differentiation Grade II-III of (Table 2), which are consistent with factors in women with breast cancer without HFrEF reported in previous studies [48-52]. Furthermore, our study is the first to demonstrate the benefits of adjuvant PMRT with contemporary RT techniques for OS, LRR, and DM in women with left-side IDC and HFrEF receiving TM. Our findings should be considered in future clinical practice and prospective clinical trials of HF and RT for breast cancer. We suggest that adjuvant PMRT is valuable to achieving better outcomes of OS, LRR, and DM in women with left-side IDC and HFrEF receiving TM.

This study has some limitations. First, because all women with left-side breast IDC and HFrEF were enrolled from an Asian population, the corresponding ethnic susceptibility compared with the non-Asian population remains unclear; hence, our results should be cautiously extrapolated to non-Asian populations. However, no evidence exists as to the differences in oncologic outcomes in Asian versus non-Asian patients with breast IDC and HFrEF receiving TM. Second, a weak point of the study that the median follow up (6.96 and 5.09 years) could be too short for evaluation the impact on breast cancer survival or late heart side effects. Third, the diagnoses of all comorbid conditions were based on ICD-10-CM codes. However, the combination of Taiwanese TCRD and National Health Insurance Research Database (NHIRD) data appears to be a valid resource for population research on cardiovascular diseases, stroke, or chronic comorbidities [56-58]. Moreover, the Taiwan Cancer Registry Administration randomly reviews charts and interviews patients to verify the accuracy of the diagnoses, and hospitals with outlier chargers or practices may be audited and subsequently be heavily penalized if any malpractice or discrepancy is detected. Accordingly, to obtain crucial information on population specificity and disease occurrence, a large-scale randomized trial comparing carefully selected patients undergoing suitable treatments is essential. Finally, the TCRD does not contain information regarding dietary habits or body mass index, which may be risk factors for mortality. Nevertheless, considering the magnitude and statistical significance of the observed effects in this study, these limitations are unlikely to affect the conclusions.

\section{Conclusions}

Adjuvant PMRT was associated with a decrease in all-cause death and LRR among women with left-side breast IDC and HFrEF compared with no adjuvant
PMRT. We suggest adjuvant PMRT for women with left-side IDC receiving TM, even if they have HFrEF.

\section{Abbreviations}

PMRT: Postmastectomy RT; LRR: Locoregional recurrence; DM: Distant metastasis; IDC: Invasive ductal carcinoma; HFrEF: Heart failure with reduced ejection fraction; TM: Total mastectomy; OS: Overall survival; aHR: Adjusted hazard ratio; HR: Hazard ratio; IPTW: Inverse probability of treatment weighting; Cl: Confidence interval; AJCC: American Joint Committee on Cancer; TCRD: Taiwan Cancer Registry Database; SD: Standard deviation; HER2: Human epidermal growth factor receptor-2; SLNB: Sentinel lymph node biopsy; ALND: Axillary lymph node dissection; CCl: Charlson comorbidity index; ICD-10-CM: International Classification of Diseases, 10th Revision, Clinical Modification; NCCN: National Comprehensive Cancer Network; RT: Radiotherapy; RICT: Radiotherapy-related cardiotoxicity; TM: Total mastectomy; HF: Heart failure; LV: Left ventricular; LVEF: Left ventricular ejection fraction; T:Tumor; N: Nodal; pT: Pathologic tumor stage; pN: Pathologic nodal stage; NHIRD: National Health Insurance Research Database.

\section{Acknowledgements}

Lo-Hsu Medical Foundation, Lotung Poh-Ai Hospital, supports Szu-Yuan Wu's work (Funding Number: 10908, 10909, 11001, 11002, 11003, 11006 , and 11013). Taipei Medical University-Wan Fang Hospital (Funding Number: 107TMU-WFH-08) supports Tsai-Mu Cheng's work.

Access to data statement: We used data from the National Health Insurance Research Database (NHIRD) and Taiwan Cancer Registry database (TCRD). The authors confirm that, for approved reasons, some access restrictions apply to the data underlying the findings. The data utilized in this study cannot be made available in the manuscript, the supplemental files, or in a public repository due to the "Personal Information Protection Act" executed by Taiwan's government, starting from 2012. Requests for data can be sent as a formal proposal to obtain approval from the ethics review committee of the appropriate governmental department in Taiwan. Specifically, links regarding contact info for which data requests may be sent to are as follows: http://nhird.nhri.org. tw/en/Data_Subsets.htmI\#S3 and http://nhis.nhri.org.tw/point.html. Szu-Yuan $\mathrm{Wu}, \mathrm{MD}, \mathrm{PhD}$ had full access to all the data in the study and takes responsibility for the integrity of the data and the accuracy of the data analysis. Role of funder statement: Lo-Hsu Medical Foundation, Lotung Poh-Ai Hospital, supports Szu-Yuan Wu's work (Funding Number: 10908, 10909, 11001, $11002,11003,11006$, and 11013) for design and conduct of the study.

\section{Authors' contributions}

Conception and design: JZ, S-YW; Financial support: Lo-Hsu Medical Foundation, Lotung Poh-Ai Hospital, supports Szu-Yuan Wu's work (Funding Number: 10908, 10909, 11001, 11002, 11003, 11006, and 11013). Taipei Medical University-Wan Fang Hospital (Funding Number: 107TMU-WFH-08) supports Tsai-Mu Cheng's work. Collection and assembly of data: JZ, S-YS, S-YW. Data analysis and interpretation: JZ, J-GH, M-FC, T-SL. Administrative support: S-YW; Manuscript writing: JZ, S-YS, M-FC, S-YW. All authors read and approved the final manuscript.

\section{Funding}

Lo-Hsu Medical Foundation, Lotung Poh-Ai Hospital, supports Szu-Yuan Wu's work (Funding Number: 10908, 10909, 11001, 11002, 11003, 11006, and 11013). Taipei Medical University-Wan Fang Hospital (Funding Number: 107TMU-WFH-08) supports Tsai-Mu Cheng's work.

\section{Availability of data and materials}

The data sets supporting the study conclusions are included in this manuscript and its supplementary files.

For software: Project name: not applicable; Project homepage: not applicable; Archived version: not applicable; Operating system(s): not applicable; Programming language: not applicable; Other requirements: not applicable; License: not applicable; Any restrictions for use by nonacademicians: not applicable. 


\section{Declarations}

Ethics approval and consent to participate

The study protocols were reviewed and approved by the Institutional Review Board of Tzu-Chi Medical Foundation (IRB109-015-B).

\section{Consent for publication}

Not applicable.

\section{Competing interests}

The authors have no potential competing interests to declare. The data sets supporting the study conclusions are included in the manuscript.

\section{Author details}

${ }^{1}$ Department of Anesthesiology and Perioperative Medicine, Henan Provincial People's Hospital, People's Hospital of Zhengzhou University, Zhengzhou, Henan, China. ${ }^{2}$ Department of General Surgery, Lo-Hsu Medical Foundation, Lotung Poh-Ai Hospital, Yilan, Taiwan. ${ }^{3}$ Graduate Institute of Business Administration, Fu Jen Catholic University, Taipei, Taiwan. ${ }^{4}$ Division of Gastroenterology and Hepatology, Department of Internal Medicine, Lo-Hsu Medical Foundation, Lotung Poh-Ai Hospital, Yilan, Taiwan. ${ }^{5}$ Department of Food Nutrition and Health Biotechnology, College of Medical and Health Science, Asia University, Taichung, Taiwan. ${ }^{6}$ Big Data \& Cancer Center, Lo-Hsu Medical Foundation, Lotung Poh-Ai Hospital, Yilan, Taiwan. ${ }^{7}$ Division of Radiation Oncology, Lo-Hsu Medical Foundation, Lotung Poh-Ai Hospital, No. 83, Nanchang St., Luodong Township, Yilan County 265, Taiwan. ${ }^{8}$ Department of Healthcare Administration, College of Medical and Health Science, Asia University, Taichung, Taiwan. ${ }^{9}$ Cancer Center, Lo-Hsu Medical Foundation, Lotung Poh-Ai Hospital, Yilan, Taiwan. ${ }^{10}$ Centers for Regional Anesthesia and Pain Medicine, Taipei Municipal Wan Fang Hospital, Taipei Medical University, Taipei, Taiwan.

Received: 18 November 2021 Accepted: 28 January 2022 Published online: 12 February 2022

\section{References}

1. Hooning MJ, Botma A, Aleman BM, Baaijens MH, Bartelink H, Klijn JG, Taylor CW, van Leeuwen FE. Long-term risk of cardiovascular disease in 10-year survivors of breast cancer. J Natl Cancer Inst. 2007;99(5):365-75.

2. Travis LB, Ng AK, Allan JM, Pui CH, Kennedy AR, Xu XG, Purdy JA, Applegate K, Yahalom J, Constine LS, et al. Second malignant neoplasms and cardiovascular disease following radiotherapy. J Natl Cancer Inst. 2012;104(5):357-70.

3. Moslehi J. The cardiovascular perils of cancer survivorship. N Engl J Med. 2013;368(11):1055-6.

4. Darby SC, Ewertz M, McGale P, Bennet AM, Blom-Goldman U, Bronnum D, Correa C, Cutter D, Gagliardi G, Gigante B, et al. Risk of ischemic heart disease in women after radiotherapy for breast cancer. N Engl J Med. 2013;368(11):987-98

5. Paszat LF, Mackillop WJ, Groome PA, Schulze K, Holowaty E. Mortality from myocardial infarction following postlumpectomy radiotherapy for breast cancer: a population-based study in Ontario, Canada. Int J Radiat Oncol Biol Phys. 1999:43(4):755-62.

6. Hojris I, Overgaard M, Christensen JJ, Overgaard J. Morbidity and mortality of ischaemic heart disease in high-risk breast-cancer patients after adjuvant postmastectomy systemic treatment with or without radiotherapy: analysis of DBCG $82 \mathrm{~b}$ and $82 \mathrm{c}$ randomised trials. Radiotherapy Committee of the Danish Breast Cancer Cooperative Group. Lancet. 1999;354(9188):1425-30.

7. Yancy CW, Jessup M, Bozkurt B, Butler J, Casey DE Jr, Drazner MH, Fonarow GC, Geraci SA, Horwich T, Januzzi JL, et al. 2013 ACCF/AHA guideline for the management of heart failure: executive summary: a report of the American College of Cardiology Foundation/American Heart Association Task Force on practice guidelines. Circulation. 2013;128(16):1810-52.

8. Ponikowski P, Voors AA, Anker SD, Bueno H, Cleland JGF, Coats AJS, Falk V, Gonzalez-Juanatey JR, Harjola VP, Jankowska EA, et al. 2016 ESC guidelines for the diagnosis and treatment of acute and chronic heart failure: the Task Force for the diagnosis and treatment of acute and chronic heart failure of the European Society of Cardiology (ESC) developed with the special contribution of the Heart Failure Association (HFA) of the ESC. Eur Heart J. 2016;37(27):2129-200.

9. Yancy CW, Jessup M, Bozkurt B, Butler J, Casey DE Jr, Colvin MM, Drazner MH, Filippatos GS, Fonarow GC, Givertz MM, et al. 2017 ACC/AHA/HFSA focused update of the 2013 ACCF/AHA guideline for the management of heart failure: a report of the American College of Cardiology/American Heart Association Task Force on Clinical Practice Guidelines and the Heart Failure Society of America. Circulation. 2017;136(6):e137-61.

10. Zhang J, Lu CY, Chen $\mathrm{CH}$, Chen HM, Wu SY. Effect of pathologic stages on postmastectomy radiation therapy in breast cancer receiving neoadjuvant chemotherapy and total mastectomy: a cancer database analysis. Breast. 2020;54:70-8.

11. Zhang J, Lu CY, Qin L, Chen HM, Wu SY. Breast-conserving surgery with or without irradiation in women with invasive ductal carcinoma of the breast receiving preoperative systemic therapy: a cohort study. Breast. 2020;54:139-47.

12. Zhang J, Lu CY, Chen HM, Wu SY. Neoadjuvant chemotherapy or endocrine therapy for invasive ductal carcinoma of the breast with high hormone receptor positivity and human epidermal growth factor receptor 2 negativity. JAMA Netw Open. 2021;4(3):e211785.

13. Liu WC, Liu HE, Kao YW, Qin L, Lin KC, Fang CY, Tsai LL, Shia BC, Wu SY. Definitive radiotherapy or surgery for early oral squamous cell carcinoma in old and very old patients: a propensity-score-matched, nationwide, population-based cohort study. Radiother Oncol. 2020;151:214-21.

14. NCCN Clinical practice guidelines in oncology. http://www.nccn.org/ professionals/physician_gls/f_guidelines.asp.

15. Hammond ME, Hayes DF, Dowsett M, Allred DC, Hagerty KL, Badve S, Fitzgibbons PL, Francis G, Goldstein NS, Hayes M, et al. American Society of Clinical Oncology/College Of American Pathologists guideline recommendations for immunohistochemical testing of estrogen and progesterone receptors in breast cancer. J Clin Oncol Off J Am Soc Clin Oncol. 2010;28(16):2784-95.

16. Charlson M, Szatrowski TP, Peterson J, Gold J. Validation of a combined comorbidity index. J Clin Epidemiol. 1994;47(11):1245-51.

17. Chen JH, Yen YC, Yang HC, Liu SH, Yuan SP, Wu LL, Lee FP, Lin KC, Lai MT, Wu CC, et al. Curative-intent aggressive treatment improves survival in elderly patients with locally advanced head and neck squamous cell carcinoma and high comorbidity index. Medicine (Baltimore). 2016;95(14):e3268.

18. Lin SH, Wang L, Myles B, Thall PF, Hofstetter WL, Swisher SG, Ajani JA, Cox JD, Komaki R, Liao Z. Propensity score-based comparison of long-term outcomes with 3-dimensional conformal radiotherapy versus intensitymodulated radiotherapy for esophageal cancer. Int J Radiat Oncol Biol Phys. 2012;84(5):1078-85.

19. Austin PC, Stuart EA. Moving towards best practice when using inverse probability of treatment weighting (IPTW) using the propensity score to estimate causal treatment effects in observational studies. Stat Med. 2015;34(28):3661-79.

20. Austin PC. Balance diagnostics for comparing the distribution of baseline covariates between treatment groups in propensity-score matched samples. Stat Med. 2009;28(25):3083-107.

21. Clarke M, Collins R, Darby S, Davies C, Elphinstone P, Evans V, Godwin J, Gray R, Hicks C, James S, et al. Effects of radiotherapy and of differences in the extent of surgery for early breast cancer on local recurrence and 15-year survival: an overview of the randomised trials. Lancet. 2005;366(9503):2087-106.

22. Danish Breast Cancer Cooperative G, Nielsen HM, Overgaard M, Grau C, Jensen AR, Overgaard J. Study of failure pattern among high-risk breast cancer patients with or without postmastectomy radiotherapy in addition to adjuvant systemic therapy: long-term results from the Danish Breast Cancer Cooperative Group DBCG $82 \mathrm{~b}$ and c randomized studies. J Clin Oncol: Off J Amer Soc Clin Oncol 2006;24(15):2268-2275.

23. Ragaz J, Olivotto IA, Spinelli JJ, Phillips N, Jackson SM, Wilson KS, Knowling MA, Coppin CM, Weir L, Gelmon K, et al. Locoregional radiation therapy in patients with high-risk breast cancer receiving adjuvant chemotherapy: 20-year results of the British Columbia randomized trial. J Natl Cancer Inst. 2005;97(2):116-26.

24. Yu JM, Hsieh MC, Qin L, Zhang J, Wu SY. Metformin reduces radiationinduced cardiac toxicity risk in patients having breast cancer. Am J Cancer Res. 2019;9(5):1017-26. 
25. Lee CH, Zhang JF, Yuan KS, Wu ATH, Wu SY. Risk of cardiotoxicity induced by adjuvant anthracycline-based chemotherapy and radiotherapy in young and old Asian women with breast cancer. Strahlenther Onkol. 2019;195:629-39.

26. Giordano SH, Kuo YF, Freeman JL, BuchholzTA, Hortobagyi GN, Goodwin JS. Risk of cardiac death after adjuvant radiotherapy for breast cancer. J Natl Cancer Inst. 2005;97(6):419-24.

27. Darby SC, McGale P, Taylor CW, Peto R. Long-term mortality from heart disease and lung cancer after radiotherapy for early breast cancer: prospective cohort study of about 300,000 women in US SEER cancer registries. Lancet Oncol. 2005;6(8):557-65.

28. Patt DA, Goodwin JS, Kuo YF, Freeman JL, Zhang DD, BuchholzTA, Hortobagyi GN, Giordano SH. Cardiac morbidity of adjuvant radiotherapy for breast cancer. J Clin Oncol Off J Am Soc Clin Oncol. 2005;23(30):7475-82.

29. Taylor CW, Bronnum D, Darby SC, Gagliardi G, Hall P, Jensen MB, McGale P, Nisbet A, Ewertz M. Cardiac dose estimates from Danish and Swedish breast cancer radiotherapy during 1977-2001. Radiother Oncol. 2011;100(2):176-83.

30. McGale P, Darby SC, Hall P, Adolfsson J, Bengtsson NO, Bennet AM, Fornander T, Gigante B, Jensen MB, Peto R, et al. Incidence of heart disease in 35,000 women treated with radiotherapy for breast cancer in Denmark and Sweden. Radiother Oncol. 2011;100(2):167-75.

31. Boekel NB, Jacobse JN, Schaapveld M, Hooning MJ, Gietema JA, Duane FK, Taylor CW, Darby SC, Hauptmann M, Seynaeve CM, et al. Cardiovascular disease incidence after internal mammary chain irradiation and anthracycline-based chemotherapy for breast cancer. Br $J$ Cancer. 2018;119(4):408-18.

32. Early Breast Cancer Trialists' Collaborative G, Peto R, Davies C, Godwin J, Gray R, Pan HC, Clarke M, Cutter D, Darby S, McGale P, et al. Comparisons between different polychemotherapy regimens for early breast cancer: meta-analyses of long-term outcome among 100,000 women in 123 randomised trials. Lancet. 2012;379(9814):432-44.

33. Blum JL, Flynn PJ, Yothers G, Asmar L, Geyer CE Jr, Jacobs SA, Robert NJ, Hopkins JO, O'Shaughnessy JA, Dang CT, et al. Anthracyclines in early breast cancer: the ABC Trials-USOR 06-090, NSABP B-46-I/USOR 07132, and NSABP B-49 (NRG Oncology). J Clin Oncol Off J Am Soc Clin Oncol. 2017;35(23):2647-55.

34. Zamorano JL, Lancellotti P, Rodriguez Munoz D, Aboyans V, Asteggiano R, Galderisi M, Habib G, Lenihan DJ, Lip GYH, Lyon AR, et al. 2016 ESC position paper on cancer treatments and cardiovascular toxicity developed under the auspices of the ESC Committee for Practice Guidelines: the Task Force for cancer treatments and cardiovascular toxicity of the European Society of Cardiology (ESC). Eur Heart J. 2016;37(36):2768-801.

35. Cardinale D, Colombo A, Bacchiani G, Tedeschi I, Meroni CA, Veglia F, Civelli M, Lamantia G, Colombo N, Curigliano G, et al. Early detection of anthracycline cardiotoxicity and improvement with heart failure therapy. Circulation. 2015;131(22):1981-8.

36. Von Hoff DD, Layard MW, Basa P, Davis HL Jr, Von Hoff AL, Rozencweig M, Muggia FM. Risk factors for doxorubicin-induced congestive heart failure. Ann Intern Med. 1979;91(5):710-7.

37. Swain SM, Whaley FS, Ewer MS. Congestive heart failure in patients treated with doxorubicin: a retrospective analysis of three trials. Cancer. 2003:97(11):2869-79.

38. Schwartz RG, McKenzie WB, Alexander J, Sager P, D'Souza A, Manatunga A, Schwartz PE, Berger HJ, Setaro J, Surkin L, et al. Congestive heart failure and left ventricular dysfunction complicating doxorubicin therapy. Seven-year experience using serial radionuclide angiocardiography. Am J Med. 1987;82(6):1109-18.

39. Keefe DL. Trastuzumab-associated cardiotoxicity. Cancer. 2002;95(7):1592-600.

40. Perez EA, Rodeheffer R. Clinical cardiac tolerability of trastuzumab. J Clin Oncol Off J Am Soc Clin Oncol. 2004;22(2):322-9.

41. Fiuza M. Cardiotoxicity associated with trastuzumab treatment of HER2+ breast cancer. Adv Ther. 2009:26(Suppl 1):S9-17.

42. Mehta LS, Watson KE, Barac A, Beckie TM, Bittner V, Cruz-Flores S, Dent S, Kondapalli L, Ky B, Okwuosa T, et al. Cardiovascular disease and breast cancer: where these entities intersect: a scientific statement from the American Heart Association. Circulation. 2018;137(8):e30-66.

43. Roger VL, Weston SA, Redfield MM, Hellermann-Homan JP, Killian J, Yawn BP, Jacobsen SJ. Trends in heart failure incidence and survival in a community-based population. JAMA. 2004;292(3):344-50.
44. Shen L, Jhund PS, Petrie MC, Claggett BL, Barlera S, Cleland JGF, Dargie HJ, Granger CB, Kjekshus J, Kober L, et al. Declining risk of sudden death in heart failure. N Engl J Med. 2017;377(1):41-51.

45. Zhang J, Sun M, Chang E, Lu CY, Chen HM, Wu SY. Pathologic response as predictor of recurrence, metastasis, and survival in breast cancer patients receiving neoadjuvant chemotherapy and total mastectomy. Am J Cancer Res. 2020;10(10):3415-27.

46. Zhang J, Qin L, Chen HM, Hsu HC, Chuang CC, Chen D, Wu SY. Overall survival, locoregional recurrence, and distant metastasis of definitive concurrent chemoradiotherapy for cervical squamous cell carcinoma and adenocarcinoma: before and after propensity score matching analysis of a cohort study. Am J Cancer Res. 2020;10(6):1808-20.

47. Zhang J, Lu CY, Chen HM, Wu SY. Pathologic response rates for breast cancer stages as a predictor of outcomes in patients receiving neoadjuvant chemotherapy followed by breast-conserving surgery. Surg Oncol. 2020;36:91-8.

48. Yoo S, Lee HB, Han W, Noh DY, Park SK, Kim WH, Kim JT. Total intravenous anesthesia versus inhalation anesthesia for breast cancer surgery: a retrospective cohort study. Anesthesiology. 2019;130(1):31-40.

49. Oh TK, Kim HH, Jeon YT. Retrospective analysis of 1-year mortality after gastric cancer surgery: total intravenous anesthesia versus volatile anesthesia. Acta Anaesthesiol Scand. 2019;63(9):1169-77.

50. Lee JH, Kang SH, Kim Y, Kim HA, Kim BS. Effects of propofol-based total intravenous anesthesia on recurrence and overall survival in patients after modified radical mastectomy: a retrospective study. Korean J Anesthesiol. 2016;69(2):126-32.

51. Enlund $M$, Berglund $A$, Ahlstrand R, Wallden J, Lundberg J, Warnberg F, Ekman A, Sjoblom Widfeldt N, Enlund A, Bergkvist L. Survival after primary breast cancer surgery following propofol or sevoflurane general anesthesia-a retrospective, multicenter, database analysis of 6305 Swedish patients. Acta Anaesthesiol Scand. 2020;64(8):1048-54.

52. Makito K, Matsui H, Fushimi K, Yasunaga H. Volatile versus total intravenous anesthesia for cancer prognosis in patients having digestive cancer surgery. Anesthesiology. 2020;133(4):764-73.

53. Fiorentino A, Gregucci F, Mazzola R, Figlia V, Ricchetti F, Sicignano G, Giajlevra N, Ruggieri R, Fersino S, Naccarato S, et al. Intensity-modulated radiotherapy and hypofractionated volumetric modulated arc therapy for elderly patients with breast cancer: comparison of acute and late toxicities. Radiol Med. 2019;124(4):309-14.

54. Meduri B, Gregucci F, D'Angelo E, Alitto AR, Ciurlia E, Desideri I, Marino L, Borghetti P, Fiore M, Fiorentino A, et al. Volume de-escalation in radiation therapy: state of the art and new perspectives. J Cancer Res Clin Oncol. 2020;146(4):909-24.

55. Fiorentino A, Mazzola R, Giaj Levra N, Fersino S, Ricchetti F, Di Paola G, Gori S, Massocco A, Alongi F. Comorbidities and intensity-modulated radiotherapy with simultaneous integrated boost in elderly breast cancer patients. Aging Clin Exp Res. 2018;30(5):533-8.

56. Cheng CL, Lee CH, Chen PS, Li YH, Lin SJ, Yang YH. Validation of acute myocardial infarction cases in the National Health Insurance Research Database in Taiwan. J Epidemiol. 2014;24(6):500-7.

57. Cheng CL, Kao YH, Lin SJ, Lee CH, Lai ML. Validation of the National Health Insurance Research Database with ischemic stroke cases in Taiwan. Pharmacoepidemiol Drug Saf. 2011;20(3):236-42.

58. Lin CC, Lai MS, Syu CY, Chang SC, Tseng FY. Accuracy of diabetes diagnosis in health insurance claims data in Taiwan. J Formos Med Assoc. 2005;104(3):157-63.

\section{Publisher's Note}

Springer Nature remains neutral with regard to jurisdictional claims in published maps and institutional affiliations. 